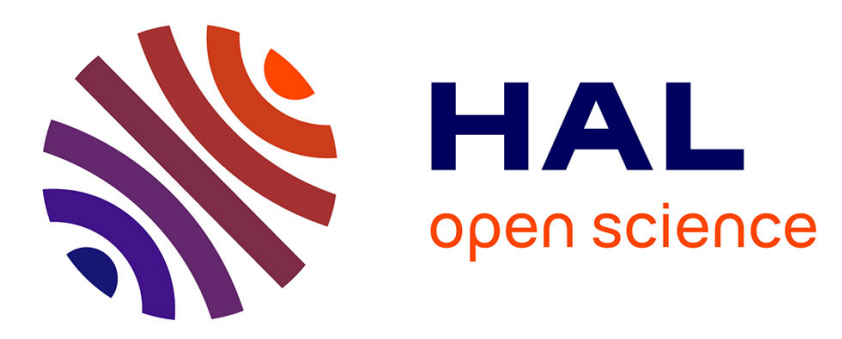

\title{
Custom implant design for large cranial defects
}

Filipe M. M. Marreiros, Yann Heuzé, Michael Verius, Claudia Unterhofer, Wolfgang Freysinger, Wolfgang Recheis

\section{To cite this version:}

Filipe M. M. Marreiros, Yann Heuzé, Michael Verius, Claudia Unterhofer, Wolfgang Freysinger, et al.. Custom implant design for large cranial defects. International Journal of Computer Assisted Radiology and Surgery, 2016, pp.14. 10.1007/s11548-016-1454-8 . hal-01842538

\section{HAL Id: hal-01842538 \\ https://hal.science/hal-01842538}

Submitted on 11 Jan 2021

HAL is a multi-disciplinary open access archive for the deposit and dissemination of scientific research documents, whether they are published or not. The documents may come from teaching and research institutions in France or abroad, or from public or private research centers.
L'archive ouverte pluridisciplinaire HAL, est destinée au dépôt et à la diffusion de documents scientifiques de niveau recherche, publiés ou non, émanant des établissements d'enseignement et de recherche français ou étrangers, des laboratoires publics ou privés. 


\title{
Custom implant design for large cranial defects
}

\author{
Filipe M. M. Marreiros • Y. Heuzé • M. \\ Verius • C. Unterhofer • W. Freysinger • \\ W. Recheis
}

Received: date / Accepted: date

\author{
Abstract Purpose \\ The aim of this work was to introduce a Computer Aided Design (CAD) \\ tool that enables the design of large skull defects $\left(>100 \mathrm{~cm}^{2}\right)$ implants. Func- \\ tional and aesthetically correct custom implants are extremely important for \\ Filipe M. M. Marreiros \\ Center for Medical Image Science and Visualization (CMIV), Linköping University, SE-581 \\ 85 Linköping, Sweden \\ Linköping University, Department of Medical and Health Sciences (IMH), Linköping Uni- \\ versity, SE-581 85 Linköping, Sweden \\ Department of Science and Technology (ITN) - Media and Information Technology (MIT), \\ Linköping University, Sweden \\ E-mail: filipe.marreiros@liu.se \\ Yann Heuzé \\ University of Bordeaux, UMR 5199 PACEA, Bordeaux Archaeological Sciences Cluster of \\ Excellence, Université de Bordeaux, Allée Geoffroy de Saint Hilaire Bat. B8, CS 50023 , \\ 33615 Pessac Cedex, France \\ E-mail: yann.heuze@u-bordeaux.fr \\ Michael Verius \\ Innsbruck Medical University, Department of Radiology, Innrain 52, Christoph-Probst-Platz, \\ 6020 Innsbruck, Austria \\ E-mail: michael.verius@i-med.ac.at \\ Claudia Unterhofer \\ Innsbruck Medical University, Clinical Department of Neurosurgery, Innrain 52, Christoph- \\ Probst-Platz, 6020 Innsbruck, Austria \\ E-mail: claudia.unterhofer@tirol-kliniken.at \\ Wolfgang Freysinger \\ Innsbruck Medical University, Department of Otorhinolaryngology (ENT), Hearing, Speech \\ and Voice Disorders, Innrain 52, Christoph-Probst-Platz, 6020 Innsbruck, Austria \\ E-mail: wolfgang.freysinger@i-med.ac.at \\ Wolfgang Recheis \\ Innsbruck Medical University, Department of Radiology, Innrain 52, Christoph-Probst-Platz, \\ 6020 Innsbruck, Austria \\ E-mail: wolfgang.recheis@i-med.ac.at
}


patients with large cranial defects. For these cases pre-operative fabrication of implants is recommended to avoid problems of donor site morbidity, sufficiency of donor material and quality. Finally, crafting the correct shape is a non-trivial task increasingly complicated by defect size.

Methods

We present a Computer Aided Design (CAD) tool to design such implants for the neurocranium. A combination of Geometric Morphometrics (GM) and Radial Basis Functions (RBF), namely Thin Plate Splines (TPS), allows semiautomatic implant generation. The method uses symmetry and the best fitting shape to estimate missing data directly within the radiologic volume data. In addition, this approach delivers correct implant fitting via a boundary fitting approach.

Results

This method generates a smooth implant surface, free of sharp edges that follows the main contours of the boundary, enabling accurate implant placement in the defect site intraoperatively. The present approach is evaluated and compared to existing methods. A mean error of $89.29 \%(72.64 \%-100 \%)$ missing landmarks with an error less or equal to $1 \mathrm{~mm}$ was obtained.

Conclusion

In conclusion, the results show that our Computer Aided Design (CAD) tool can generate patient specific implants with high accuracy.

Keywords Cranial reconstruction · reconstructive surgery · geometric morphometrics $\cdot$ radial basis functions and thin plate spline

\section{Introduction}

Functional and aesthetically correct custom implants are extremely important for patients with large cranial defects $\left(>100 \mathrm{~cm}^{2}\right)$. Usually, small defects are treated using autograft material due to the high rate of a successful incorporation by defect site tissues. However, problems of donor site morbidity, sufficient donor material, bone tissue quality and crafting the correct shape from the available donor increase dramatically with the size of the defect. In these cases the pre-operative fabrication of an implant should be considered.

Using current medical imaging modalities, in our case 3D Computed Tomography (CT) and specialized software tools the anatomically correct design of such implants is possible [1, [2, 3], 4, [5], 6], 7], 8], [9, 10, 11, 12, 13 14, 15. The different software tools vary in the user interaction required to design the implants: manual, semi-automatic or automatic. Semi-automatic or automatic methods aid considerably the design of such implants and a complete and coherent software CAD (Computer Aided Design) pipeline is of great importance to the user in charge of the production of the implants.

In the next sections we will present the pipeline blocks and methods of our CAD implant design tool that allows the generation of $3 \mathrm{D}$ virtual implants that can be further used to produce the physical implants. 


\section{Materials and methods}

Several CAD tools exist that can be used for implant design ranging from manual to automatic solutions. The core of our CAD tool is a semi-automatic method that relies on the interpolation properties of the Radial Basis Function (RBF), in particular the Thin Plate Spline (TPS), and the statistical tools of Geometric Morphometrics (GM) [16], [17]. The GM methods belong to the more general statistical shape analysis methods, which is the analysis of the geometrical properties of sets of shapes by statistical methods. An introduction to these methods is provided in 18. Before we describe in detail the blocks that compose the $\mathrm{CAD}$ tool pipeline we will introduce the most important methods and compare to related work. All the defects presented in this work, including simulated defects used for evaluation are large skull defects $\left(>100 \mathrm{~cm}^{2}\right)$.

\subsection{Radial Basis Function}

RBFs can be used as surface interpolators, the reconstruction properties of the RBFs have been described in detail by Carr et al. 14, [19; the present work is inspired by these methods, and builds upon our previous work 20. RBFs are used in GM to study shape intersubject differences using deformation grids. In Computer Graphics (CG) RBFs are mainly used for interpolation purposes to generate smooth functions passing through input data (centers) and can be used, for instance, for hole filling.

In GM however, RBFs are used to encode differences between shapes, or to map one shape onto another. TPS warping and spline relaxation are among the most used applications of RBFs in GM.

The highly complex structure of the human skull in the facial area exhibits sharp edges and holes so that the presented method can only be used for the neurocranium, where TPS can be employed to minimize bending energy [21.

\subsection{GM - Landmark}

A detailed landmark classification can be found in the work of Bookstein 16. In brief, semilandmarks are points on a structure allowing the incorporation of information from ridge curves and surfaces of the structure, i.e. a skull. Classical anatomical landmarks bear full threedimensional information, whereas semilandmarks present "deficient" coordinates and are computed on base of the available coordinates. Semilandmarks are treated as missing data and are estimated in order to minimize the bending energy between a template shape and the target shape. 2D semilandmarks and spline relaxation along curves were introduced by Bookstein [22], work that was extended to three dimensions by Gunz et al. 23. A curve, surface or fully relaxed semilandmark is a landmark with one, two or three unknown dimensions and thus semilandmarks can slide on curves, surfaces and in 3D space. Anatomical landmarks are not relaxed, they are fully defined. 
Fully relaxed semilandmarks are generally used to estimate semilandmarks of regions where missing data occur, i. e. defect sites. We will not differentiate between landmarks and semilandmarks, both will be distinguished by relaxation type: anatomical, curve relaxed, surface relaxed and fully relaxed landmark. Missing landmarks are any of the previous ones that are used to delineate the defect region for implant placement with a slight overlap to correctly compute the implant boundary. In most cases the complete set of missing landmarks is formed by surface relaxed and fully relaxed landmarks.

\subsection{GM - methods used}

From the suite of GM methods we use the Procrustes Average Shape (PAS), TPS spline relaxation and Generalized Procrustes Analysis (GPA) 24. The PAS is computed using a database of 130 anonymized CT exams with axial slices and average slice thickness of $1 \mathrm{~mm}$. Demographically, 73 males (average age 51.14 years, standard deviation 16.28 years, maximum 82 years, minimum 21 years) and 57 females (average age 57.26 years, standard deviation 21.32 years, maximum 97 years, minimum 20 years) are in the data set. PAS and GPA align the population of shapes iteratively and are well known [23, 20]. In every iteration each specimen is relaxed to the PAS and is aligned using GPA. With all shapes aligned and relaxed we compute the PAS for the actual iteration. The final PAS should be taken from the final iteration.

The results of this operation are: the PAS (that might happen to be the best fitting shape) and all landmark sets in the database relaxed to the PAS. To compute the best fitting shape we also make use of GPA, for more details see sections: measuring the anatomical landmarks, initial landmark estimation and fully relaxed landmark selection. The fully relaxed landmarks are obtained using symmetry information (if possible) and the best fitting shape.

\subsection{Related work}

The closest work to ours is by Min and Dean [8, 9], 10, who also use landmarks and a TPS warp 22 for the reconstruction. Despite a basically similar concept, our approach differs particularly with respect to the determination of the defect boundary, implant triangle mesh generation and the number of specimens that provide the estimate for missing landmarks. The present approach, however, goes beyond their work by incorporating symmetry and the best fitting shape instead of either symmetry or mean shape. We also used the TPS spline relaxation instead of the TPS warp providing a more accurate positioning of the landmarks, as explained later on in the text. The last main difference is that we developed a method to find the best fitting shape from our database; in this paper we will show that using the best fitting shape combined with symmetry is in most cases superior to just using a mean shape.

The main difference to Carr's surface interpolation with RBFs for medical imaging [14, is that the missing landmarks and centers of the RBF, of the 
defect site are obtained by GM methods. Carr's surface interpolation uses the vertices of the defect's boundary region as the centers of the RBFs.

$\mathrm{Wu}$ et al. [6] used anatomical constrained deformations based on reference models (3D-matching and adaptive deformation) and compared their method against RBFs. They found that RBFs provided worse results as only the boundary information was used as centers for the RBFs.

Finaly, Lüthi et al. [5] showed that Procrustes alignment is used to generate a statistical shape model using probabilistic principal component analysis and Liao et al. 2, 4 4 used active contours and image registration for the reconstruction. These methods can be used also to estimate missing data; however, they do not allow to incorporate symmetry information, one of the key sources of information of our method.

\subsection{CAD design pipeline}

The CAD tool is built upon the Visualization Toolkit (VTK) 25] and the design pipeline is shown in Figure 1. The process starts by performing a manual threshold segmentation (a value of 250 was used in all cases) of the 3D CT DICOM data. Since the region of interest is the largest connected bony region, all small bone fragments and artifacts have to be removed.

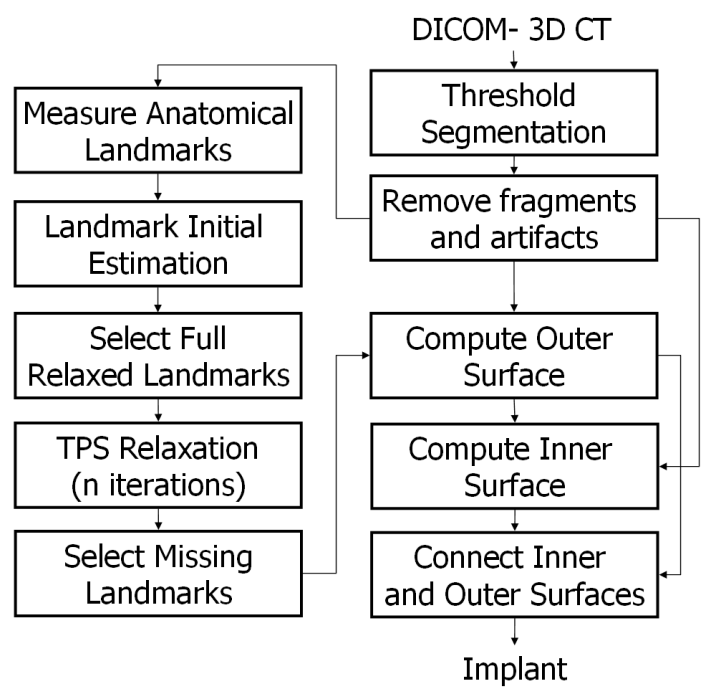

Fig. 1 Implant design pipeline.

The next step is to manually measure the anatomical landmarks. Followed by an initial estimate of all remaining landmark positions. In the defect region the fully relaxed landmarks are estimated using the TPS relaxation. After $n$ relaxation iterations the missing landmarks are manually selected and used 
for generating the outer surface mesh of the implant. The outer mesh serves to calculate the mesh of the implant's inner surface. Furthermore the surface has to be fitted to the defect boundary. This implies identifying the triangles of the defect boundary and finally to connect both the inner and the outer meshes to the final implant mesh.

\subsubsection{Removal of artifacts and fragments}

Artifacts and fragments removal is done with the vtkImageSeedConnectivity, which is a connected component labeling algorithm [26, [27]. Since we know that the CTs contain only skulls, and that the largest connected region in the (multiplanar reformatted) medial sagittal plane corresponds to the skull and not a fragment or an artifact, we can on this slice test every voxel above threshold for its connectivity check the largest connectivity region. The pixels (actually voxels) with the largest connected region are selected for a 3D volume connectivity test. Finally, the largest connected region is kept and all other removed.

\subsubsection{Measuring the anatomical landmarks, initial landmark estimation and} fully relaxed landmark selection

17 cranial anatomical landmarks are measured; some are standard landmarks for GM studies [28], 29], all are listed in Table 1. Two sets of standard landmarks are non-standard: $(8,9)$ and $(12,13)$, were introduced to form a more complete landmark distribution around the skull.

Table 1 Anatomical landmarks used in this study.

\begin{tabular}{cc}
\hline \hline IDs & Names \\
\hline 1 & Nasion \\
2,3 & Frontomalare Orbitale. (right, left) \\
4,5 & Frontomalare Temporale. (right, left) \\
6,7 & Fronto Temporale. (right, left) \\
8,9 & The most medial point in the sphenoid \\
& bone that is parallel to the frontomalare \\
& orbitale when the skull is viewed \\
& laterally. (right, left) \\
10,11 & Zygomatic Root (right, left) \\
12,13 & The point in the Zygomatic Process \\
& of the temporal bone most \\
14 & anterior medial. (right, left) \\
15 & Opisthion \\
16,17 & Masion \\
& Maxillo Frontale (right, left)
\end{tabular}

A ray-casting approach [30, 31, 32 was used to measure/set the landmarks via a trilinear interpolated, ray sampled at $0.05 \mathrm{~mm}$. Figure 2 shows some of the anatomical landmarks on the skull. An initial estimate of the 
semilandmarks was obtained applying different projection schemes, by using different sets of anatomical landmarks (according to the anatomical location) to generate projection planes or lines where rays are casted to the surface.
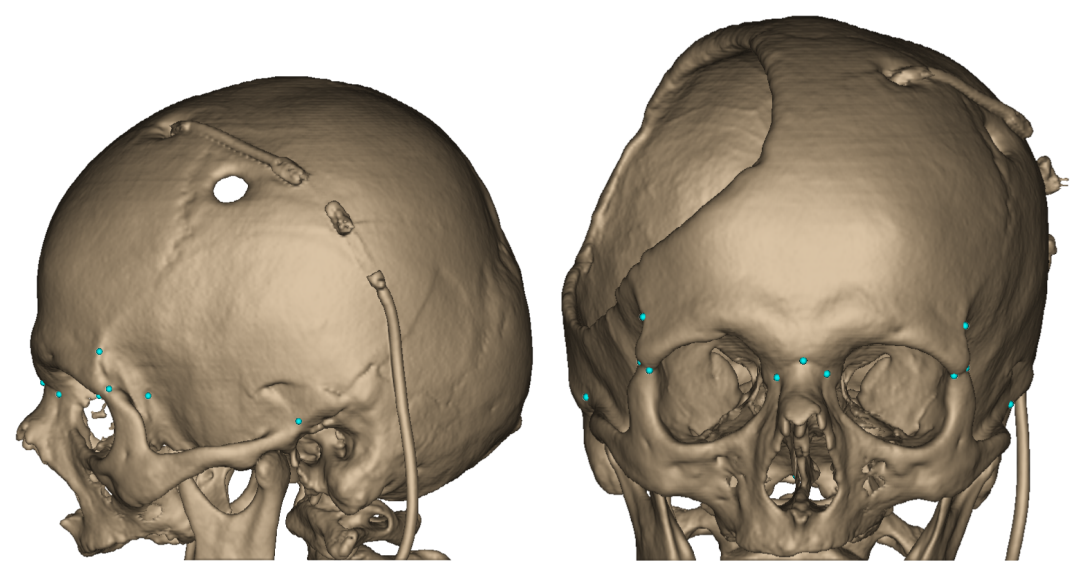

Fig. 2 Anatomical Landmarks (cyan) as seen from different views. The tubular structure is a catheter for ventricular drainage and is shown as it has a Hounsfield units similar to bone.

This is exemplified on one of these regions, the region defined by the bilateral landmarks Fronto Temporale and Zygomatic Root. These landmarks define a plane that allows defining circular sampling lines perpendicular to it with the respective center at midline, defined by the line connecting the centroids of the two pairs of bilateral landmarks. For demonstration purposes a few sampling and initialization points on the boundary (green and blue points, respectively) are shown. This ray-casting is defined via the projection centers along the midline (red points in Figure 3) and the corresponding circular sampling points (green dots in Figure 3). Corresponding (surface relaxed) landmarks are generated if the ray reaches the last voxel above or equal to a threshold; else the landmark is considered fully relaxed. The initial estimates for a complete skull (727 landmarks) are shown in Figure 4. Missing anatomical landmarks need to be estimated for defective skulls. All missing landmarks can be estimated via symmetry, or else via the best fitting shape (defined below). To estimate these anatomical landmarks via symmetry we first calculate the side (left or right) of the skull that has most fully relaxed landmarks, this will be defined as target and the opposite side as the template. The bilateral missing landmarks are excluded. The template set will contain all the defined landmarks of the corresponding side while the target set the symmetric points, if these are missing they are set to fully relaxed. In this case the landmarks do not slide (explained below - only one iteration is required). After the TPS relaxation the fully relaxed landmarks will hold the new landmark positions. This procedure is repeated twice, for each side of the skull. For the bilateral missing landmarks the best fitting shape is used, by setting the missing land- 
marks to fully relaxed and perform TPS relaxation to obtain their position. Once the anatomical landmarks are known the remaining landmarks can also be obtained in a similar fashion.

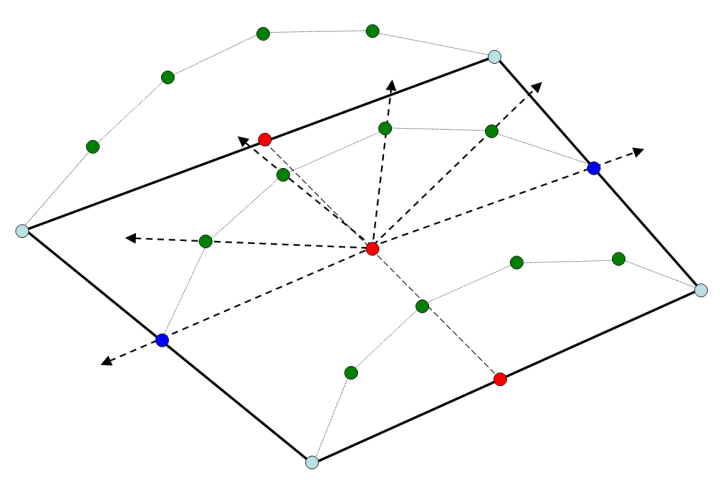

Fig. 3 Projection scheme: The solid line represents the plane formed by the 4 anatomical bilateral landmarks Fronto Temporale and Zigomatic Root (light blue). The dark blue points are sampling positions along the lateral boundaries used to define the semicircles orthogonal to the plane. The green points are sampling positions along the semicircles, the red points are sampled in the midline. The projection is started at the red points radially in the direction of the sampling points (arrows). The rays stop at the last voxel above or equal to threshold, providing the new landmark positions.

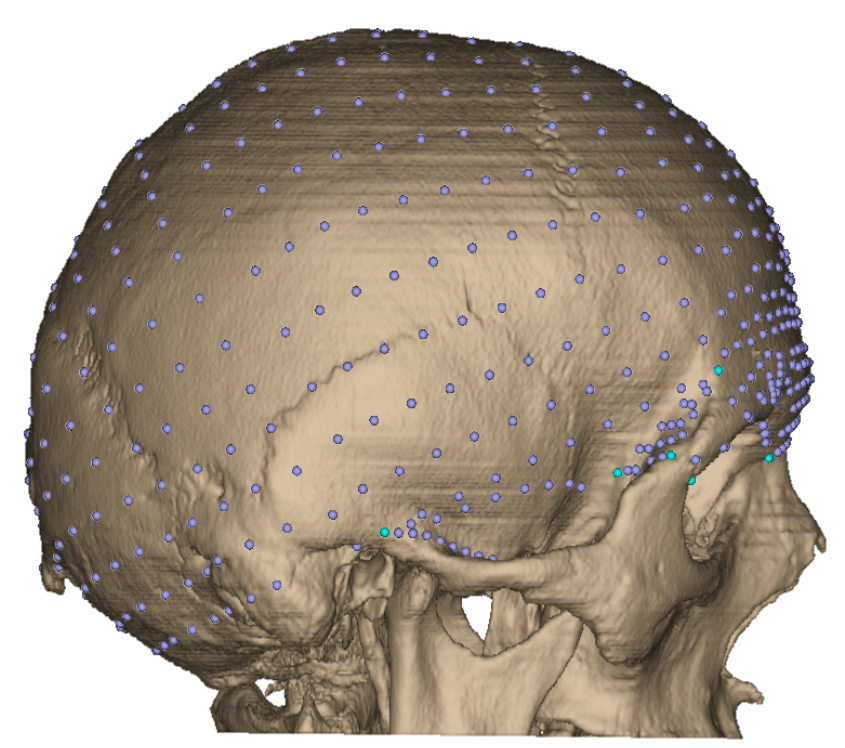

Fig. 4 Landmarks placed on the skull surface using the initial estimation method (727 landmarks). The initial estimation uses anatomical landmarks (cyan) to define projection schemes used to place the surface landmarks (violet). 
In the symmetry case the TPS is used considering that the available landmarks (surface landmarks) are not relaxed, they do not slide, because surface normals are pointing generally in opposite directions for bilateral landmarks, this means that the tangent planes are not equivalent consequently the sliding is not possible. Once we obtain the landmark estimation of one side we have to repeat the operation for the remaining side.

The best fitting shape is obtained via GPA alignment of the patient shape and all relaxed shapes in the database including the PAS without considering the fully relaxed landmarks. The set (patient shape, database shape or PAS) with the smallest Root Mean Square Error (RMSE) between the landmark positions of the patient shape and each shape in the database is taken as the best fitting shape.

Some landmarks still exist that have to be set manually, c. f. Figure 5. e. g. at the inner surface of the skull or at the boundary region of the defect.

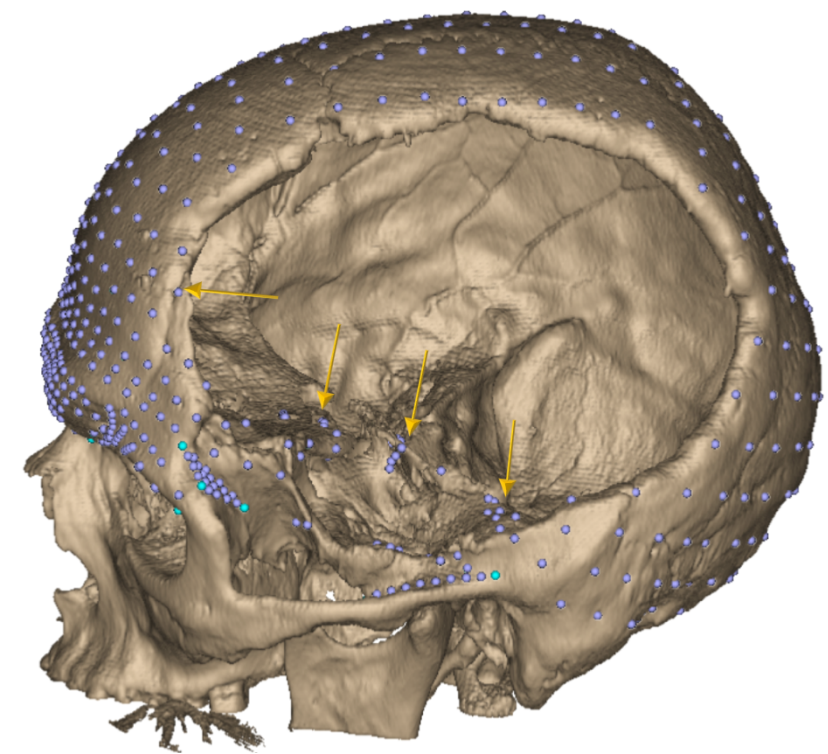

Fig. 5 Initial landmark estimation of a defective skull. Landmarks on the inner surface of the skull and on the boundary region of the defect need to be selected manually to full relaxation (arrows point to some of these landmarks).

\subsubsection{TPS relaxation}

The TPS relaxation is used to obtain the PAS, to estimate missing data and to slide surface landmarks to positions with minimum bending energy. All skulls in the database are complete (with no defect) with anatomical and surface landmarks. For the relaxation calculations the anatomical landmarks are considered as surface landmarks. Setting anatomical landmarks to surface 
relaxed landmarks will allow sliding all the landmarks to positions providing minimal bending energy between target and template shapes. Moreover, user subjectivity in landmark measurement is eliminated. Some TPS generated landmarks might be slightly misplaced with respect to the correct anatomical sites (mainly due to the user initial setting); however, they are guaranteed to be in homologous positions.

For analyzing defective skull data the anatomical landmarks are also set to surface relaxed landmarks. All available surface landmarks are used and the best fitting shape is iteratively searched.

GM is frequently performed with TPS relaxation on triangular meshes, but never, as far as we know, was the voxel information used directly. The process is started by computing the surface relaxation tangent plane were the landmarks can slide. The normal of the plane can be computed with a 3D extension of the 2D Sobel operator [33] that uses trilinear interpolation to generate isotropic data. The plane can be defined using the normal and the landmark position. Some tangent planes and the normal vectors at the landmarks of a complete skull are displayed in Figure [6

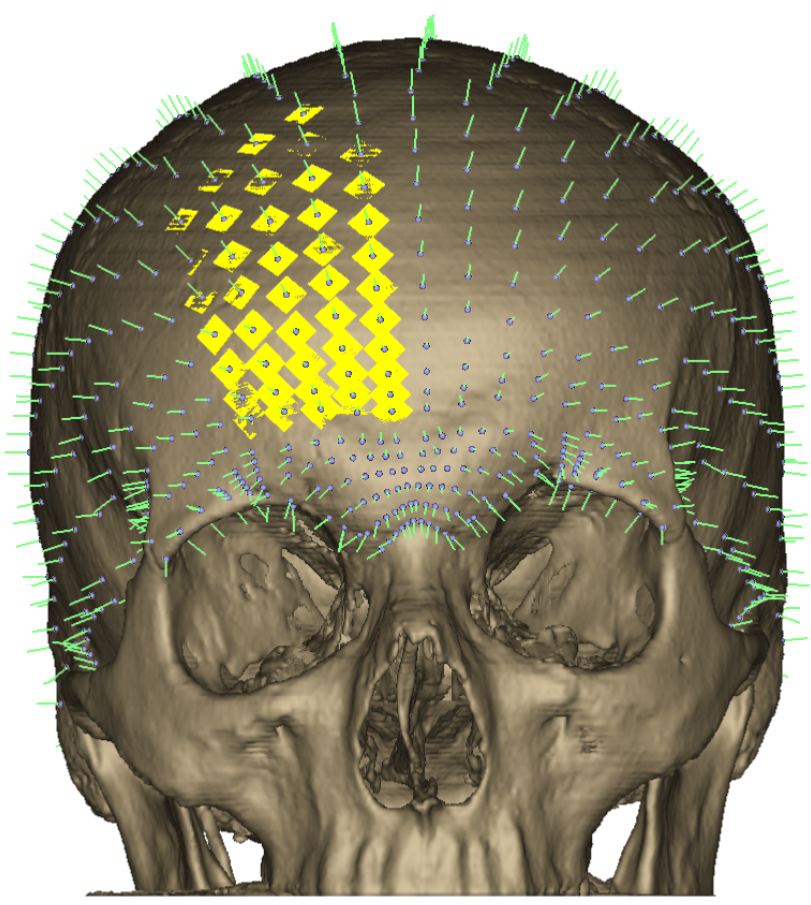

Fig. 6 Normals (green lines) of the local tangent planes (yellow) for each landmark.

Landmark relaxation slides the landmarks on the tangent planes so that they eventually move off the anatomic surface under consideration and need to be projected back on the surface. It is essential for the whole process that 
this projection generates landmarks on the outer surface. This is assured by sampling around the landmarks in a 101x101x101 voxels bounding box to classify whether voxels are in the outer surface or not: its value has to be at least the bone threshold and at least one of its 26 neighbors has to be below threshold. All other possibilities are classified as non-boundary voxels, ideally providing two sets of voxels corresponding to inner and outer surfaces, respectively. For the following, the outer surface only is considered. The new landmark position will correspond to the voxel with the smallest distance to the previous landmark position.

\subsubsection{Exterior surface interpolation using $2 D R B F$}

The interpolation of the exterior surface is done with a 2D RBF [14] that allows the construction of a depth-map centered at the (manually selected) missing landmarks of the defective skull. In order to close the implant boundary to the rest of the rest of the defective skull, some of the missing landmarks are placed on the bone, see later.

The depth-map requires a plane that allows the correct implant reconstruction. This plane is defined by the normal vector chosen as the actual direction of the surgical implant insertion process and a point at the centroid of the missing landmarks. One point of this vector is defined as the centroid of all the landmarks, the other is the centroid of the missing landmarks. The exterior surface (Figure 7) is obtained by evaluating the depth-map at points corresponding to the vertices of the mesh on a regular triangle grid $(z=0)$ with adaptable numbers of columns and rows in order to create triangles greater than the largest neighbor voxel distance.

\subsubsection{Triangle classification and connectivity test}

The connectivity test is crucial for implant design. The mesh is sampled regularly along the triangle surface and interpolated trilinearly using its eight neighbor voxels. Each triangle is labeled:

"1" In space: All sampling gray values are below the threshold.

"2" Inside the bone: All sampling gray values are above the threshold.

"3" Else: Mixed.

All triangles with the label "2" intercept the outer surface of the skull and can be discarded. The implant corresponds to the largest connected region formed by triangles with label 1(cyan region, Figure 8) that can be chosen simply by counting its triangles on the regular grid.

In figure (Figure 8) the two possible test configurations [34] with a maximum of twelve connected neighbors (gray triangles) are presented, the configuration to be used is dependent on the triangle type: upper or lower triangle.

This test, similar to the connected components labeling algorithm [26], 27, is applied to the triangles, if no neighbor triangles share the value, a new group is formed. If only one shares the label, it is added to this labeled group; if more 


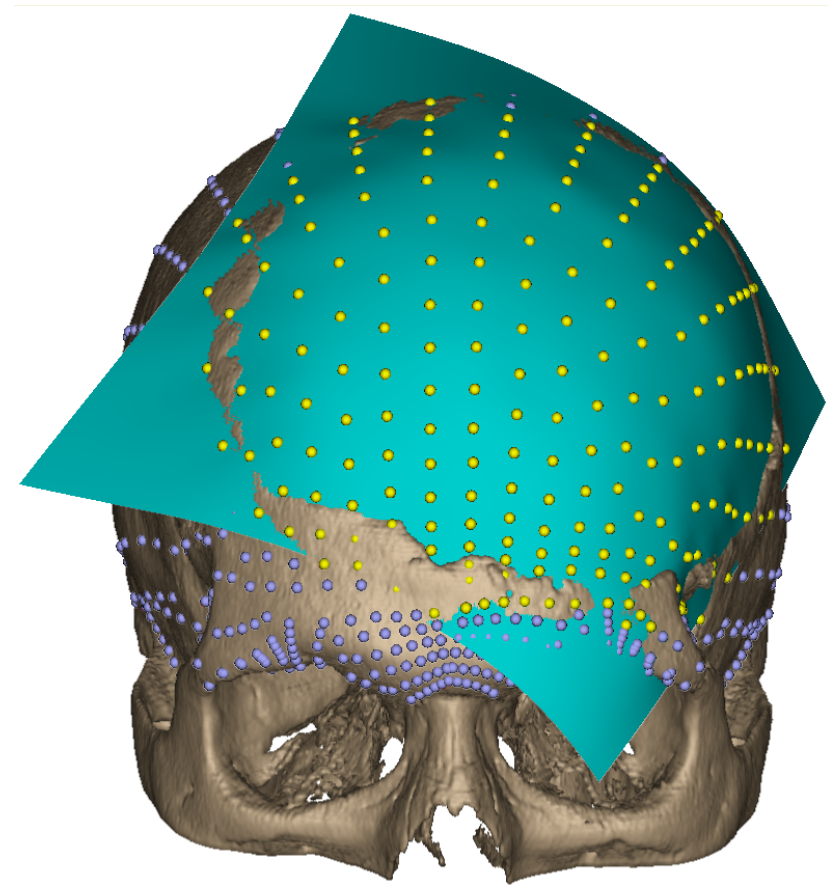

Fig. 7 Exterior mesh obtained using the 2D RBF. The yellow points are missing landmarks, the other points surface landmarks, the surface is shown in cyan.

than one connected triangles share the same label a new group is created and groups are merged.

\subsubsection{Boundary fitting}

The boundary triangles (red triangles, Figure 8) that have value "3" are directly connected to the triangles of the largest connected region. Triangles sharing only one edge with (i) the remaining boundary triangles and (ii) the triangles of the largest connected region are removed and now all boundary triangles have at least two connecting edges, and the fitting process can be initialized.

In order to fit the implant to the defect region, it is necessary to ensure that its boundary triangles are completely outside the bone. The process starts by independently sampling along the inner edges of border triangles of the implant as depicted in Figure 10 (top). The sampling is stopped when a sampling position below threshold (outside of bone) is found; at the previous step there is still some overlap (Figure 10, top right). Next, the border edge is sampled Figure 10 (bottom, left), if the edge has "inside sampling points" then the edge get discarded and the triangle vertices of the new edge are updated, this process is repeated until one edge with only "outside sampling points" is found. Furthermore, in order to produce a small gap between bone and 


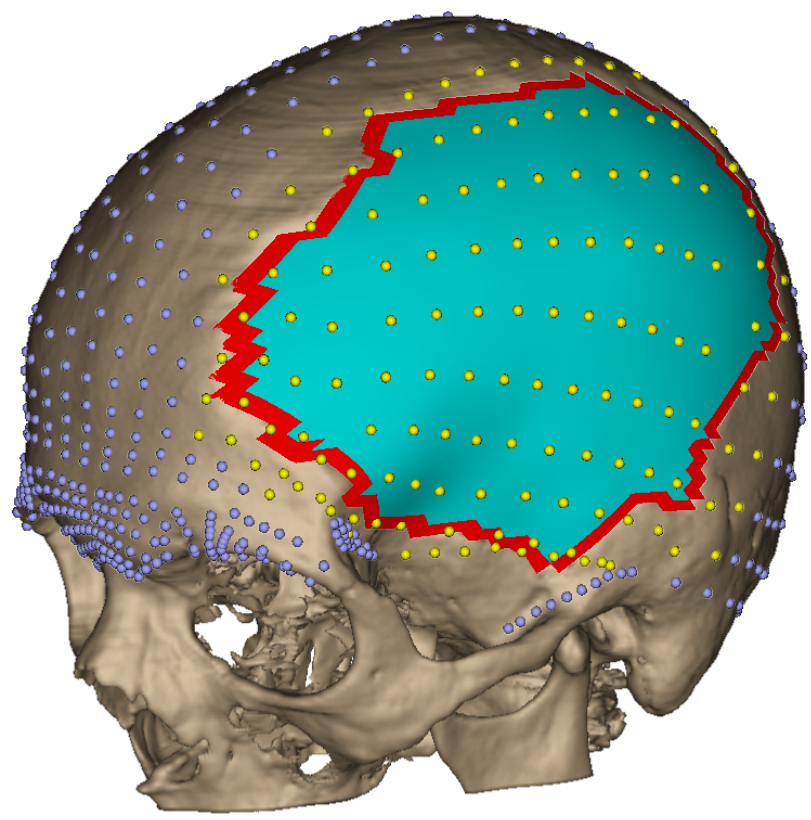

Fig. 8 Outer surface mesh as the largest connected region (selection of triangles with value " 1 ". The red triangles are directly connected to the cyan region and have value " 3 " (boundary triangles). The yellow points and violet points are fully relaxed and surface relaxed landmarks, respectively.
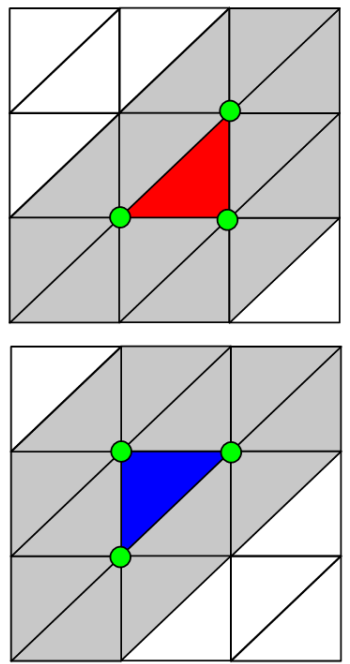

Fig. 9 The two test configurations used for triangle connectivity, depending on the tupe of triangle tested: upper (bottom) or lower (top) triangle. The green circles are the corresponding vertices used to search for the connected neighbor triangles (gray). 

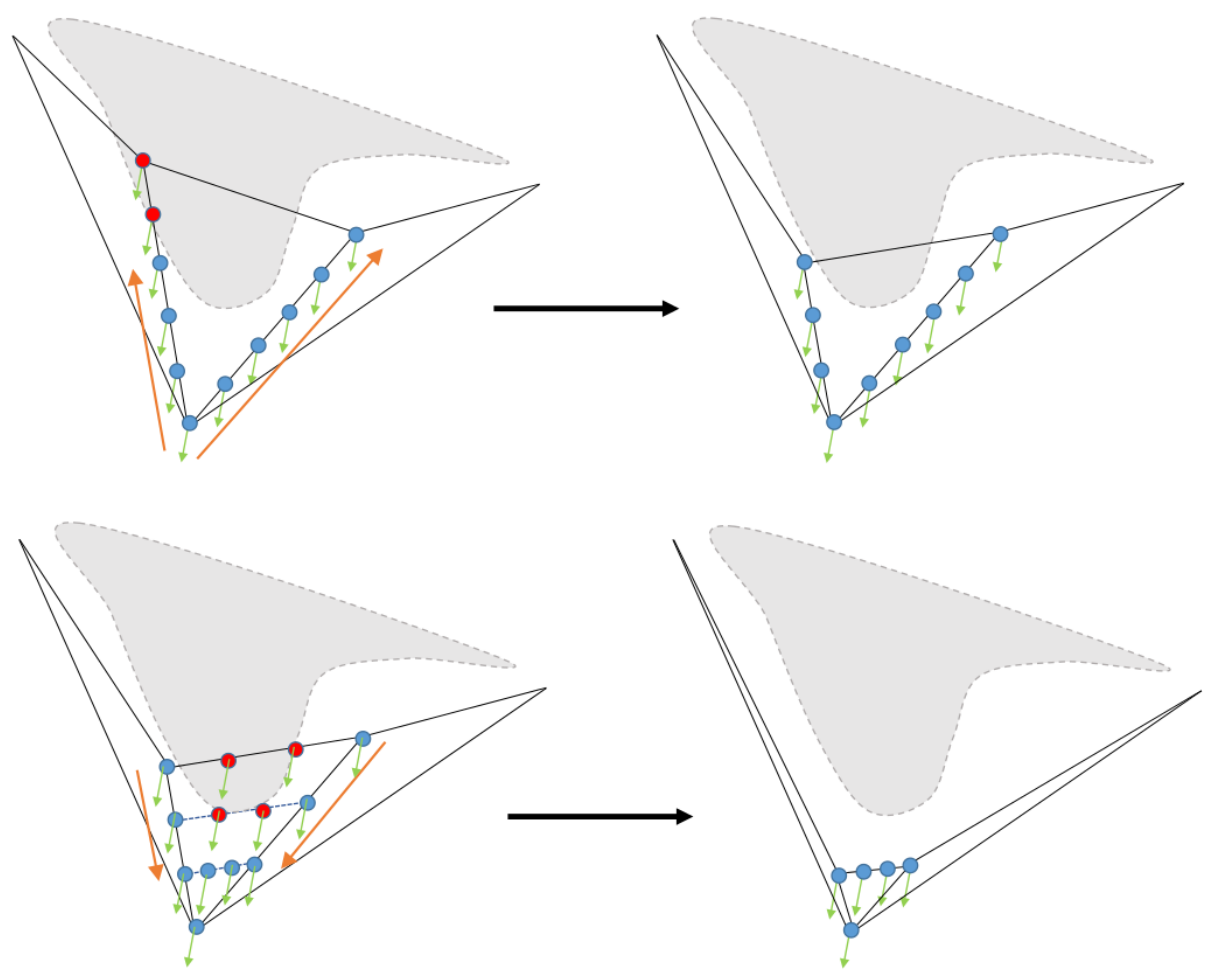

Fig. 10 Placement of the boundary triangles outside the bone. (Top) Sampling along the connected edges. (Bottom) Sampling the non connected edge (several iterations might be performed, updating the edge position, until an edge containing only outside sampling points is found). The gray area represents the bone defect area, the red dots represent the sampling points inside, and the blue dots represent the points outside. The orange arrows represent the direction of the edges sampling and the small green arrows represent the sampling performed with a direction parallel to the implant vector, this is used to provide a small gap between bone and implant, in our case a maximum distance of $1 \mathrm{~mm}$ from the origin sampling point was used. If the sampling positions along these directions contain inside sampling points then the correspondent origin sampling point is considered also an inside sampling point, otherwise an outside sampling point.

implant, sampling was performed with directions parallel to the implant vector (Figure 10-green arrows), in our case a maximum distance of $1 \mathrm{~mm}$ from the origin sampling point was used, but this can be chosen freely to control the gap size that allows accounting for accumulated errors and ease intraoperative placement. If the sampling positions along these directions contain "inside sampling points" then the correspondent origin sampling point is considered also an "inside sampling point", otherwise an "outside sampling point". 


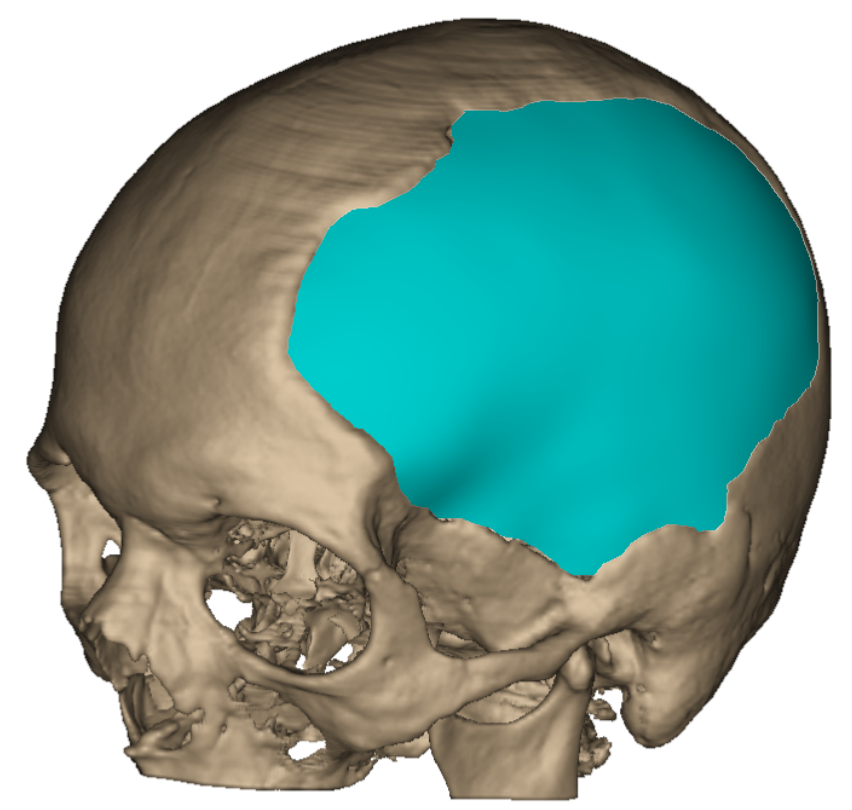

Fig. 11 Exterior implant surface after boundary fitting.

2.5.7 Creating the implant interior surface and its connection onto the exterior surface

To correctly represent the inner profile of the boundary region a much higher triangle density than on the outer surface is needed.

Figure 12 shows the two-dimensional sketch of the effects of different sampling rates. The inner surface of the implant does not resemble the outer surface of the bone at locations where the implant should fit: red lines (implant) are seen to intersect the bone. This can be attributed to insufficient sampling [35], 36]. We use a triangle subdivision scheme [37] for the outer surface that stops if a maximum set of divisions or if all triangles edges are shorter than the minimum voxel edge distance. The implant outer surface vertices are ray-casted towards the bone surface along rays parallel to the implant vector direction Figure [12, similar to [30, 31, 32]. A maximum implant thickness can be set. For safety purposes the rays are pushed back by $0.2 \mathrm{~mm}$ from their stopping points.

The last step is to connect inner and outer surfaces. First the outer boundary edge is broken up to match the number of vertices on the inner surface with the help of the previous process and the vertices are connected correctly (Figure 13). 


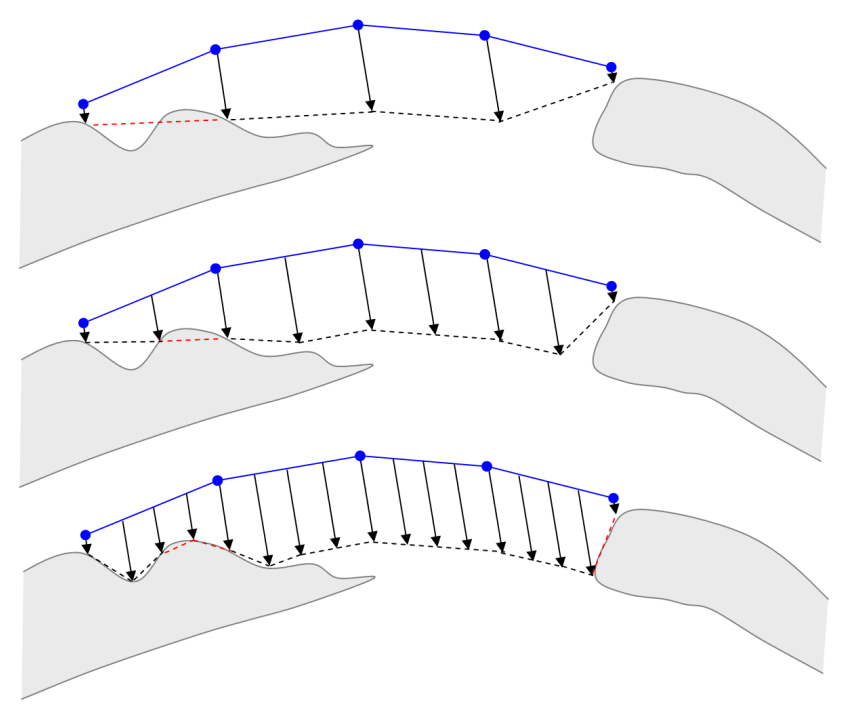

Fig. 12 Projection of the outer surface at different sampling rates to obtain the inner surface.

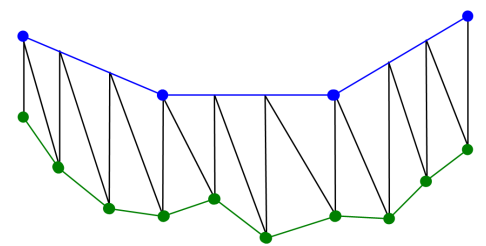

Fig. 13 Connecting inner (green) and outer (blue) surfaces.

\subsection{Evaluation}

The TPS spline relaxation is known to converge delivering sets of landmarks that minimize bending energy between the shapes involved. We used the evolution of the RMSE between iterations as an indicator of convergence. This test was applied to all skulls in the database, obtaining a combined RMSE of all possible landmark sets. And also the RMSE values for one complete skull wich is not from the database, one skull with an unilateral defect (but with all anatomical landmarks) and one skull with an bilateral defect (three anatomical landmarks are missing). The PAS was not tested because it depends on the alignment of all shapes in the database producing a slightly different PAS pose at every iteration. Since all shapes of the database are being tested the PAS test would be redundant, because if all shapes converge also the mean shape should converge.

The next test was an evaluation of the landmark position after relaxation, using four different approaches, namely testing symmetry, best fitting shape and PAS. The combinations are: 


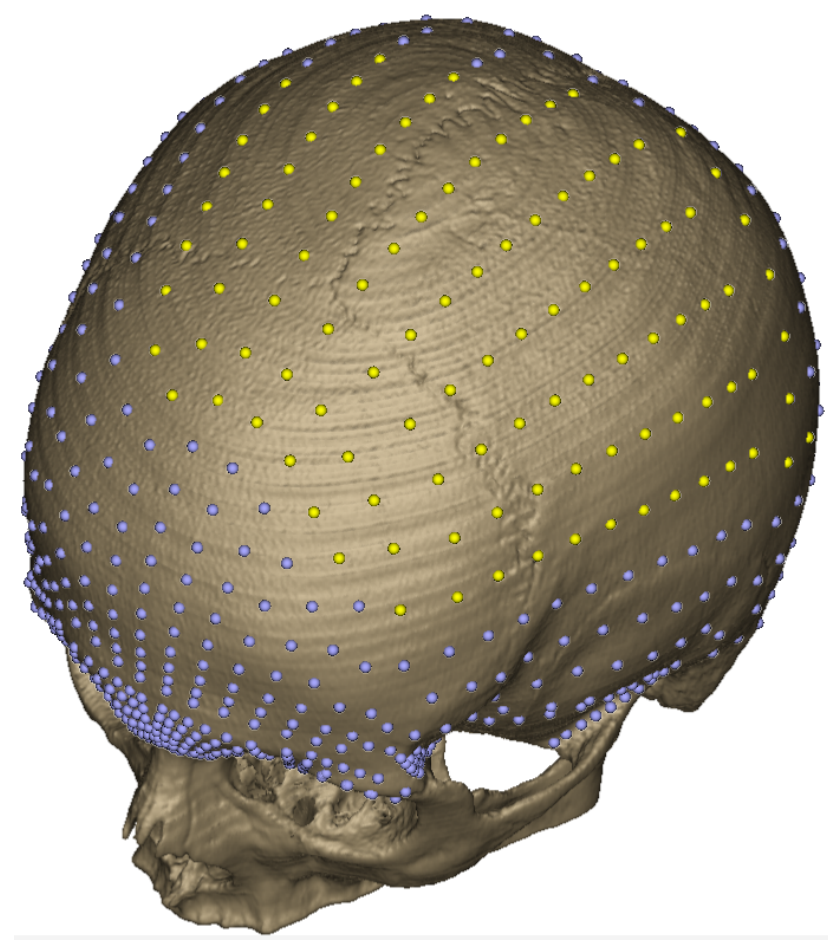

Fig. 14 Artificial skull defect (replacing surface landmarks (violet points) by fully relaxed landmarks (yellow points) and performing 10 TPS relaxation iterations). The region covered by the artificial defect involve the parietal and occipital bones.

1. Symmetry and best fitting shape

2. Symmetry and PAS

3. No symmetry only best fitting shape

4. No symmetry only PAS

Five complete skulls, not contained in the database were used. Surface landmarks were manually selected and converted to fully relaxed landmarks at different regions of the skull to simulate defects. Ideally these landmarks should be placed on the skull surface after relaxation. We computed the mean error $(\mu)$, maximum error $(\max )$ and percentage of landmarks with error less or equal to $1 \mathrm{~mm}$. The last metric was chosen because the most similar previous work have used it, enabling a comparison. The errors were calculated for fully relaxed landmarks only, and were measured as the distance from the estimated positions to the closest point on the exterior skull surface (ground truth). Ten iterations of the TPS relaxation were performed. Region A includes the parietal and occipital bones; region $\mathrm{B}$ the frontal, sphenoid and temporal bones; region $\mathrm{C}$ the frontal and parietal bones; region $\mathrm{D}$ the frontal bone(including one orbit) and finally region $\mathrm{E}$ the frontal bone (including both orbits). An example of one artificial skull defect (yellow landmarks are fully relaxed) is shown in Figure 14 corresponding to Specimen 2 region A. 


\section{Results}

The results of the convergence test can be seen in Table 2. In all cases after approximately 10 iterations the RMSE drops to considerable small values, thus the method is converging correctly, with the calculated landmark sets properly representing the shape of the skull region under consideration, and 10 iterations seem to be sufficient.

Table 2 Landmark convergence

\begin{tabular}{c|c|c|c|c}
\hline \hline Iteration & $\begin{array}{c}\text { Combined RMSE } \\
\text { of all skulls in } \\
\text { the database }(\mathrm{mm})\end{array}$ & $\begin{array}{c}\text { RMSE of complete } \\
\text { skull not in } \\
\text { the database }(\mathrm{mm})\end{array}$ & $\begin{array}{c}\text { RMSE of skull } \\
\text { with unilateral } \\
\text { defect }(\mathrm{mm})\end{array}$ & $\begin{array}{c}\text { RMSE of skull } \\
\text { with bilateral } \\
\text { defect }(\mathrm{mm})\end{array}$ \\
\hline $1-0$ & 3.08 & 3.04 & - & - \\
$2-1$ & 1.35 & 1.20 & 1.00 & 1.68 \\
$3-2$ & 0.80 & 0.83 & 0.57 & 1.04 \\
$4-3$ & 0.51 & 0.64 & 0.31 & 0.65 \\
$5-4$ & 0.37 & 0.35 & 0.22 & 0.44 \\
$6-5$ & 0.30 & 0.69 & 0.17 & 0.29 \\
$7-6$ & 0.26 & 0.59 & 0.11 & 0.26 \\
$8-7$ & 0.23 & 0.47 & 0.11 & 0.22 \\
$9-8$ & 0.22 & 0.39 & 0.10 & 0.25 \\
$10-9$ & 0.20 & 0.30 & 0.14 & 0.24 \\
$11-10$ & 0.19 & 0.18 & 0.10 & 0.24 \\
$12-11$ & 0.19 & 0.15 & 0.09 & 0.21 \\
$13-12$ & 0.19 & 0.12 & 0.08 & 0.18 \\
$14-13$ & 0.18 & 0.13 & & 0.20 \\
$15-14$ & 0.17 & 0.11 & & \\
\hline
\end{tabular}

The results of the landmark position evaluation, after relaxation are presented in Figure 15. The overall means are presented in Table 3. From the results we can observe that when using symmetry information a higher accuracy was achieved, but there was no significant difference between using the Best Fitting Shape or the PAS. The values of the four tests presented, prove the robustness of this method, even in the worst case (no symmetry only PAS - Region E, Specimen 3) a mean error of $1.01 \mathrm{~mm}$, maximum error $4.39 \mathrm{~mm}$, and $66.17 \%$ of the fully relaxed landmarks have an error less or equal to $1 \mathrm{~mm}$.

Table 3 Overall means of the: mean error $(\bar{\mu})$, maximum error $(\overline{\max })$ and percentage of landmarks with error ( $\overline{\leq 1} \mathrm{~mm})$, using the four different methods.

\begin{tabular}{c|c|c|c}
\hline \hline & $\bar{\mu}(\mathrm{mm})$ & $\leq 1(\%)$ & $\overline{\max (\mathrm{mm})}$ \\
\hline Symmetry and Best Fitting Shape & 0.42 & 89.29 & 1.79 \\
\hline Symmetry and PAS & 0.42 & 89.93 & 1.74 \\
\hline No Symmetry only Best Fitting Shape & 0.53 & 83.68 & 2.26 \\
\hline No symmetry only PAS & 0.52 & 85.05 & 2.24 \\
\hline \hline
\end{tabular}


$\mu(\mathrm{mm})$

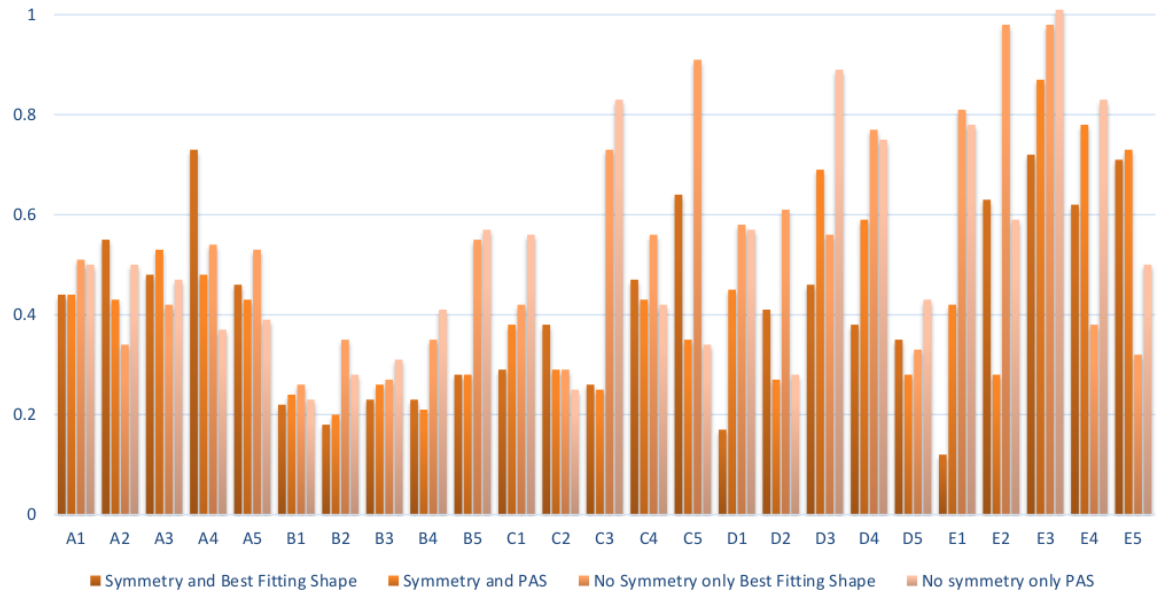

$\max (\mathrm{mm})$

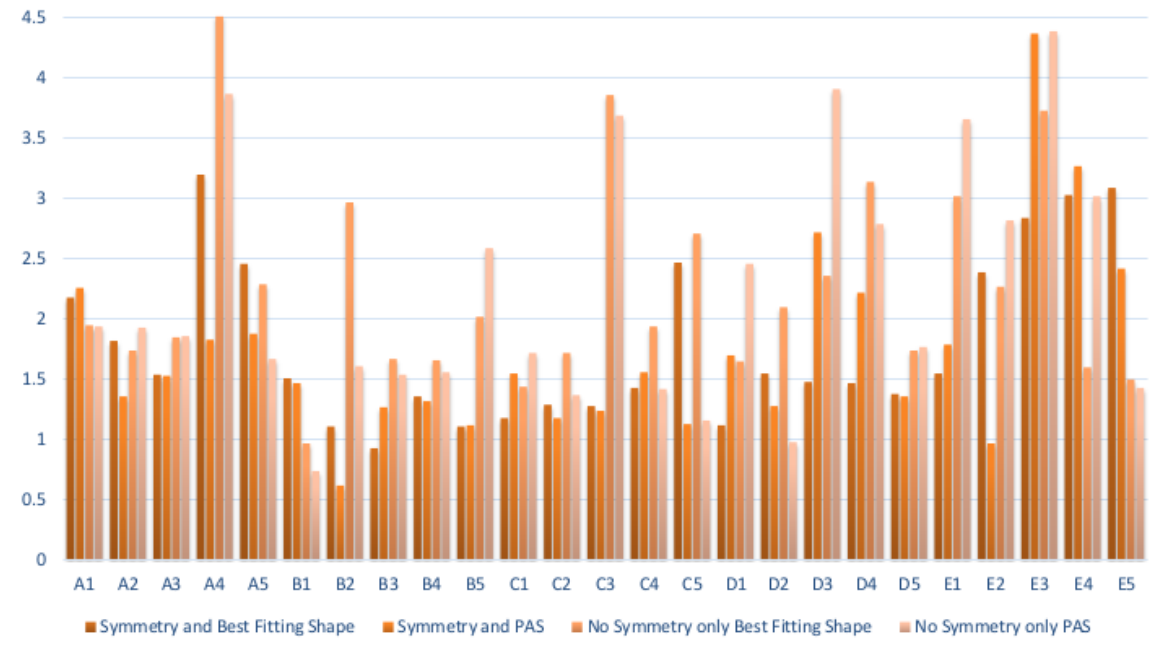

$\leq 1 \mathrm{~mm}(\%)$

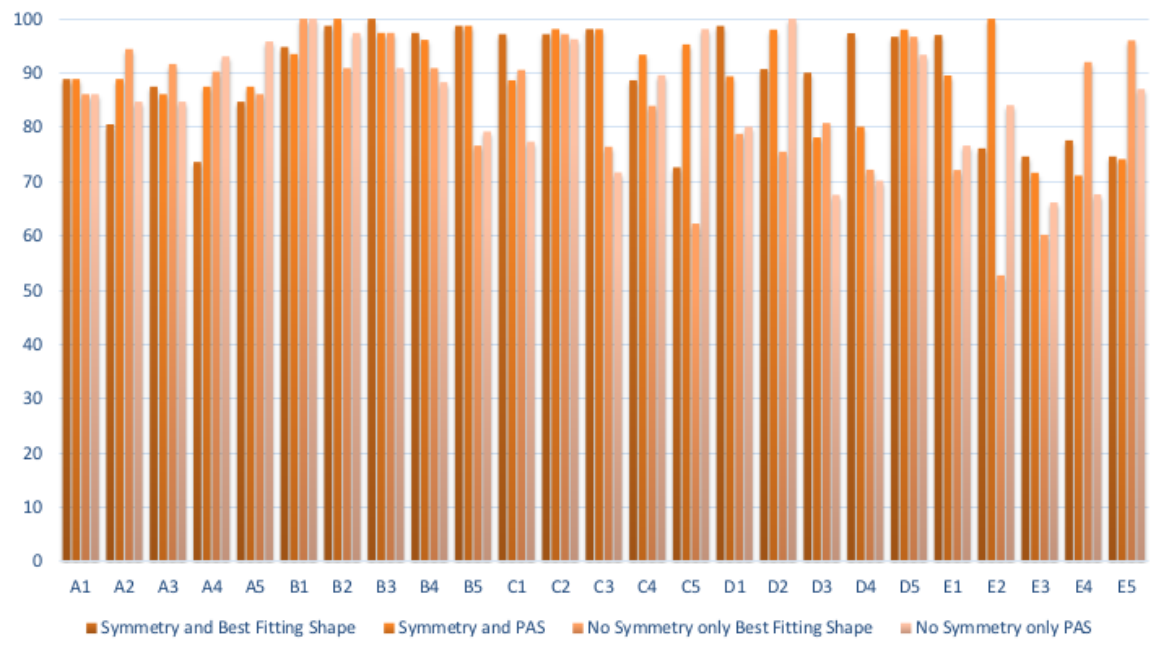

Fig. 15 Distance from estimated positions to the closest point on the exterior skull surface: Mean error $(\mu)$, maximum error $(\max )$, percentage of landmarks with error $(\leq 1 \mathrm{~mm})$. The calculations were performed with the results of the four tests: symmetry and best fitting shape, symmetry and PAS, no symmetry only best fitting shape and no symmetry only PAS. Ten iterations of the TPS relaxation were performed. Regions: A: parietal and occipital bones: B: frontal, sphenoid and temporal bones; C: frontal and parietal region; D: frontal region including one orbit; $\mathrm{E}$ : frontal region including both orbits. Five different specimens were tested. In the graphs (A1) corresponds to region A, specimen 1, the remaining cases follow the same convention. 
From all the cases studied the maximum mean error was approximately 1 $\mathrm{mm}$, a value satisfactory for the clinical problem we are considering.

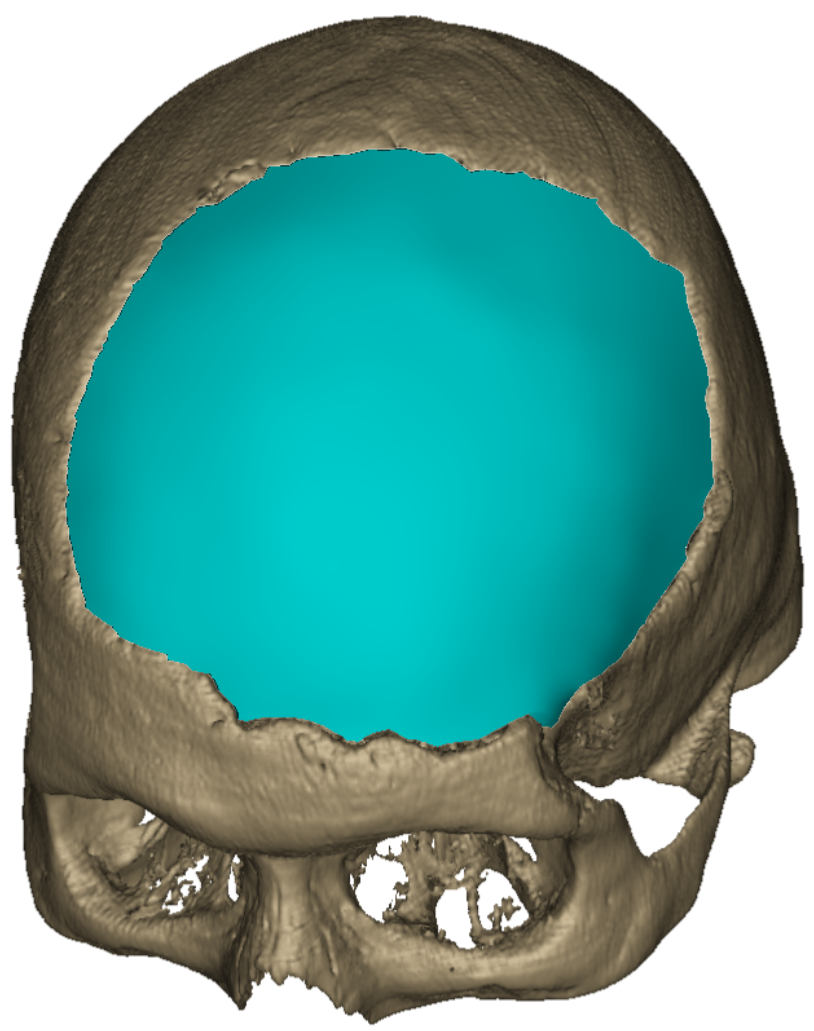

Fig. 16 Bilateral defect implant.

Two examples of implants designed by our software are presented in Figure 16 and Figure 17. An implant for a bilateral defect and a unilateral defect are shown. In Figure 17 the Skull and implant are clipped axially to allow optimal visualization of surface fitting.

\section{Discussion}

The TPS methods require landmarks that are initially placed in anatomical locations. TPS relaxation is used to regularize the landmark positions by sliding them on the surface of the skull and using the bending energy to guide the relaxation. After the relaxation the landmark positions can be compared to the landmarks of the skulls in the database. As shown in Table 2 the landmark sets within a few relaxation iterations have minimum variation between iterations and the calculated landmark sets properly represent the shape of the 


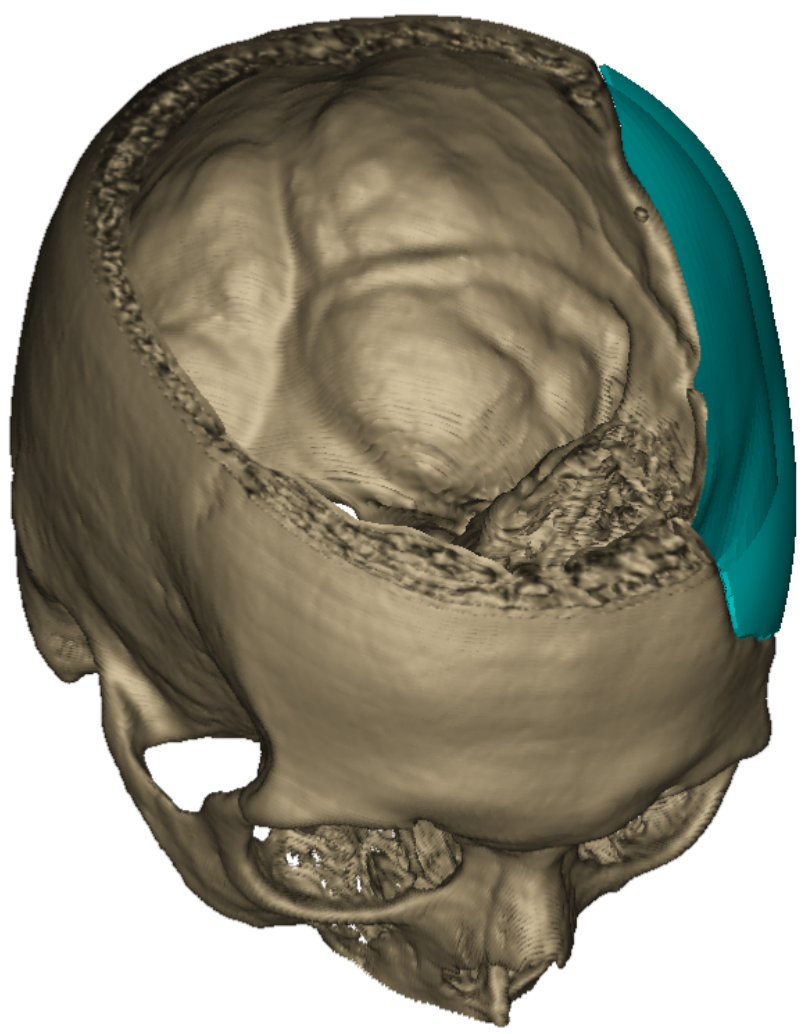

Fig. 17 Unilateral defect implant. Axially clipped to allow visualizing surface fitting.

skull region under consideration. The small differences that are still present in the last iterations can mainly be attributed to CT resolution affecting the surface tangents.

The values of the four tests presented in Figure 15, prove the robustness of this method, even in the worst case (no symmetry only PAS - Region E (frontal bone), Specimen 3) a mean error of $1.01 \mathrm{~mm}$, maximum error 4.39 $\mathrm{mm}$, and $66.17 \%$ of the fully relaxed landmarks with an error less or equal to $1 \mathrm{~mm}$ is found. Some regions provide better estimations, region A (parietal and occipital bones) and region $\mathrm{E}$ (frontal bone) are worst. The reason region A produces less accurate results is due to a smaller density of landmarks in comparison to other regions; this should be corrected in the future. Regarding region $\mathrm{E}$ it is expected to be the most difficult region to estimate [38.

It can be expected that symmetry and the best fitting shape provide the best solutions. But as Table 3 shows the values obtained are almost similar to using symmetry and PAS. This might be due to a suboptimal population of our database. Our results proof that symmetry provides better estimates. The complete skulls used for the evaluation process were not particularly asymmetrical, they are representative for the normal population, though exceptions 
could of course exist especially when the patient suffered trauma or pathologies producing some levels of asymmetry. Our method is able to compensate to some extent for asymmetric cases because it incorporates information of the landmarks surrounding the defect, this way constraining the process to obtain the missing landmarks. Furthermore, for the symmetry cases there are always two sets of points Source and Target (from opposite sides of the skull - each landmark is labeled either left of right). The missing landmarks are estimated using the TPS that uses the least bending energy to deform these shapes, i.e. a combination of information from both sides is used. This is in fact the main difference between our method and traditional symmetry methods that typically only use information from the opposite side of the defect and try to fit it to the defect side.

Wu et. al [6] reported an average of $81.6 \%$ (68\% - 96\%) surface points with an error less than 1 voxel. In our case using symmetry and the best fitting shape an average of $89.29 \%(72.64 \%$ - $100 \%)$ missing landmarks with an error less or equal to $1 \mathrm{~mm}$ was obtained. Wu's method used a mixture of small and large defects and reported for the large defects an average of $80.5 \%$ surface points within 1 voxel error.

When compared to symmetry methods like (mirrored volume) our method can look more complicated. From a user perspective with our method the user is required to identify anatomical landmarks (17 points) and once the landmark initial projections have been performed it is also necessary to select the missing landmarks; while in the mirror volume techniques the user must try to fit the mirrored volume to the defect area and most likely perform several manual post processing steps before the implant shape is finally obtained. It is crucial here to highlight the user-dependent dimension of such manual post processing and the related poor reproducibility and repeatability of such method, not to mention the time required to perform such adjustments. Though this manual approach might look less complex, we think that this approach can reveal itself as complex and time consuming without providing systematic robust results. Ultimately, if the defect is bilateral the mirror volume cannot be used.

The computation of the implant vector could be performed using alternative methods for irregularly distributed landmarks. But the present approach is simple and suffices for the majority of cases and allows for manual correction.

The boundary fitting method is not intended to produce the most accurate approximation. The goal, instead, is to obtain a smooth boundary free from sharp edges that follows the main contours of the boundary with a surgical safe margin.

All applied methods were designed for the 2D RBF case, including the connectivity optimizations. One might argue to use 3D RBFs instead [19], 39 but for the majority of cases investigated 2D RBFs are sufficient. Only for cases where ambiguities in the depth-map occur, most likely to happen at orbital regions, 3D RBFs will be the method of choice. Another advantage of the $3 \mathrm{D}$ RBFs is that missing landmarks do not have to be selected as the $3 \mathrm{D}$ RBFs provide a signed distance measure for testing whether each point (voxel) 
is inside, outside or on the boundary of the volume. With this signed distance measure all points can be classified.

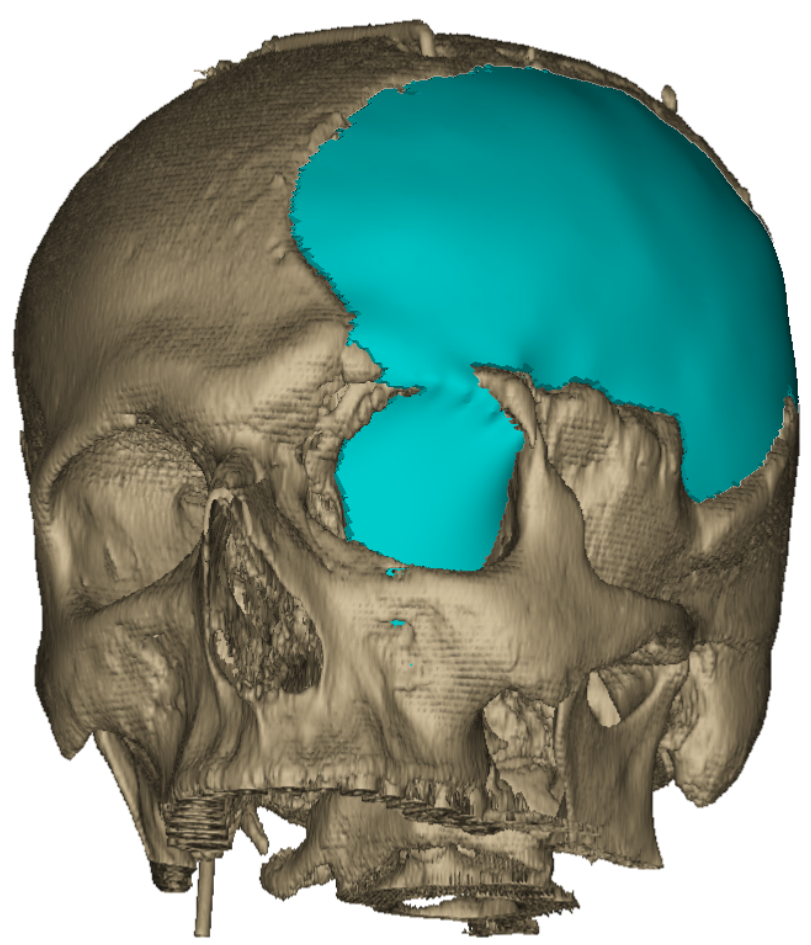

Fig. 18 Reconstruction limitation. A part of the frontal bone forming the top of the orbit is missing resulting in a leaking effect during the connectivity test.

At this time, this method has two limitations. First, if the defect area is not or is insufficiently covered by the landmark set, the reconstruction process will not be ideal and may possibly fail. Second, if a leaking effect occurs during the connectivity test due to empty regions. Figure 18 shows such a case (the frontal bone forming the top of the orbit is part of the defect area). This problem can possibly be overcome by using 3D RBFs.

The present approach, however, is simple and computationally cheap and can also be used to reconstruct other bony regions with smooth surfaces such as the femur. In an anthropological context the method could be valuable for reconstructing fragmentary bone remains. 


\section{Conclusion}

An original and innovative method for constructing implants to cover large unior bilateral defects to the neurocranium is presented by combining symmetry and best fitting shape.

The whole design process can be used as a CAD software that allows producing model files of the implant for fabrication.

Acknowledgements The authors would like to thank Brenda Frazier for editorial suggestions. We would like also to thank Philipp Gunz and Demetrios Halazonetis for their explanations of GM methods used in this work.

This work was supported by the EU FP6 Marie Curie Actions EVAN, contract number: MRTN-CT-2005-019564.

\section{Conflict of interest}

The authors declare that they have no conflict of interest.

\section{Ethical approval}

For this type of study formal consent is not required, since all the patient data was anonymized.

\section{References}

1. Parthasarathy J, (2014) 3D modeling, custom implants and its future perspectives in craniofacial surgery, Annals of Maxillofacial Surgery, 4(1):9-18.

2. Liao YL, Lu CF, Wu CT, Lee JD, Lee ST, Sun YN, Wu YT, (2013) Using threedimensional multigrid-based snake and multiresolution image registration for reconstruction of cranial defect. Medical and Biological Engineering and Computing, 51(19):89-101.

3. Rotaru H, Stan H, Florian IS, Schumacher R, Park YT, Kim SG, Chezan H, Balc N, Baciut M, (2012) Cranioplasty With Custom-Made Implants: Analyzing the Cases of 10 Patients, Journal of Oral and Maxillofacial Surgery, 70(2):169-176.

4. Liao YL, Lu CF, Sun YN, Wu CT, Lee JD, Lee ST, Wu YT, (2011) Three-dimensional reconstruction of cranial defect using active contour model and image registration. Medical and Biological Engineering and Computing, 49(2):203-211.

5. Lüthi M, Albrecht T, Vetter T, (2009) Building shape models from lousy data, MICCAI LNCS, 5762: 1-8.

6. Wu T, Engelhardt M, Fieten L, Popovic A, Radermacher K, (2006) Anatomically constrained deformation for design of cranial implant: Methodology and validation, MICCAI LNCS, 4190:9-16.

7. Hierl T, Wollny G, Schulze FP, Scholz E, Schmidt JG, Berti G, Hendricks J, Hemprich A., (2006) CAD-CAM implants in esthetic and reconstructive craniofacial surgery, Journal of Computing and Information Technology, 1:65-70.

8. Dean D., Min KJ, Bond A, (2003) Computer aided design of pre-fabricated cranial plates, Journal of Craniofacial Surgery 14:819-832.

9. Min KJ, Dean D, (2003), Highly accurate CAD tools for cranial implants, MICCAI LNCS, 2878:99-107.

10. Min KJ, (2003) Computer Aided Design of Cranial Implants Using Deformable Templates, PhD thesis, Case Western Reserve University, Cleveland Ohio. 
11. Eufinger H, Saylor B, (2001) Computer-assisted prefabrication of individual craniofacial implants, AORN Journal 74:648-654.

12. Hsu J, Tseng C, (2001) Application of three-dimensional orthogonal neural network to craniomaxillary reconstruction, Computerized Medical Imaging and Graphics, 25:477-482.

13. Hsu J, Tseng C, (2000) Application of three-dimensional orthogonal neural network to craniomaxillary reconstruction, Journal of Medical Engineering \& Technology, 24:262-266.

14. Carr JC, Fright WR, Beatson RK, (1997) Surface interpolation with radial basis functions for medical imaging, IEEE Transactions on Medical Imaging 16:96-107.

15. Wehmöller M, Eufinger H, Kruse D, Massberg W (1995), CAD by processing of computed tomography data and CAM of individually designed prostheses, International Journal of Oral Maxillofacial Surgery,24:90-7.

16. Bookstein FL, (1991) Morphometric Tools for Landmark Data: Geometry and Biology, Cambridge University Press, 1991.

17. Slice DE,(2005) Modern Morphometrics in Physical Anthropology, New York: Kluwer Academic-Prenum Publishers.

18. Stegmann MB, Gomez DD, (2002) A Brief Introduction to Statistical Shape Analysis, technical report, Informatics and Mathematical Modelling, Technical University of Denmark, DTU.

19. Carr JC, Beatson RK, Cherrie JB, Mitchell TJ, Fright WR, McCallum BC, Evans TR, (2001) Reconstruction and representation of 3D objects with radial basis functions. Computer Graphics (SIGGRAPH 01 Conf. Proc.), :67-76.

20. Heuzé Y, Marreiros FMM, Verius M, Eder R, Huttary R, Recheis W, (2008) The use of procrustes average shape in the design of custom implant surface for large skull defects, International Journal of Computer Assisted Radiology and Surgery, 3(1):283-284.

21. Bookstein FL, (1989) Principal warps: Thin-plate splines and the decomposition of deformations, IEEE Transactions on Pattern Analysis and Machine Intelligence, 6:567585 .

22. Bookstein FL, (1997) Landmark methods for forms without landmarks: morphometrics of group differences in outline shape, Medical Image Analysis, 1:225-243.

23. Gunz P, Mitteroecker P, Bookstein FL (2005) Semilandmarks in three dimensions. In: Modern Morphometrics in Physical Anthropology, Kluwer Academic/Plenum Publishers, New York, :73-98

24. Rohlf FJ, Slice D,(1990) Extensions of the procrustes method for the optimal superimposition of landmarks, Systematic Zoology, 39:40-59.

25. Schroeder W, Martin K, Lorensen B, (1997) The Visualization Toolkit: An ObjectOriented Approach to 3D Graphics, Prentice Hall.

26. Rosenfeld A, Pfaltz JL, (1966) Sequential operations in digital picture processing, Journal of the ACM 13(4):471-494.

27. Shapiro LG, Stockman GC, (2002) Computer Vision, Prentice Hall.

28. Moore-Jansen PH, Ousely SD, Jantz R, (1994) Data Collection Procedures for Forensic Skeletal Material, University of Tennessee Forensic Anthropology Series.

29. Aeillo L, Dean C, (1990) An introduction to human evolutionary anatomy, London: Academic Press, 1990.

30. Drebin RA, Carpenter L, Hanrahan P, (1988) Volume rendering. Computer Graphics $22(4): 65-74$.

31. Levoy M, (1988) Display of surfaces from volume data, IEEE Computer Graphics and Applications 8(3):29-37.

32. Levoy M, (1990), Efficient ray tracing of volume data, ACM Transactions on Graphics 9:245-261.

33. Sobel I, Feldman G, (1968) A 3 x 3 isotropic gradient operator for image processing.

34. Deutsch ES, (1972) Thinning algorithms on rectangular, hexagonal, and triangular arrays, Communications of the ACM, 15:827-837.

35. Nyquist H, (1928) Certain topics in telegraph transmission theory, Transactions of the American Institute of Electrical Engineers, 47:617-644.

36. Shannon CE, (1949) Communication in the presence of noise, Proceedings of the IRE, $37(1): 10-21$.

37. Jin X, Sun H, Peng Q, (2003) Subdivision interpolating implicit surfaces, Computer \& Graphics, 27:763-772. 
38. Poukens J, Laeven P, Beerens M, Nijenhuis G, Sloten JV, Stoelinga P, Kessler P, (2008) A classification of cranial implants based on the degree of difficulty in computer design and manufacture, International Journal of Medical Robotics and Computer Assisted Surgery, 4:46-50.

39. Turk G, O'Brien JF, (2002) Modelling with implicit sufaces that interpolate, ACM Transactions on Graphics, 21:855-873. 